\title{
Ion acoustic wave experiments in a high school plasma physics laboratory
}

Walter Gekelman

Department of Physics and Astronomy, University of California, Los Angeles, California 90095-1696

J. Wise

New Roads School, Santa Monica, California 90404

P. Pribyl

Department of Physics and Astronomy, University of California, Los Angeles, California 90095

R. Baker

University High School, Los Angeles, California 90025

W. Layton

Department of Physics and Astronomy, University of California, Los Angeles, California 90095-1696

J. Skrzypek ${ }^{\text {a) }}$

California Institute of Technology, Pasadena, California 91125

P. Niknejadi ${ }^{\text {b) }}$

California State Polytechnic University, Pomona, California 91768

R. Ransom

University of California, Los Angeles, California 90095

D. Lee

Palos Verdes Peninsula High, Palos Verdes, California 90274

R. Zarinshesnas

University High School, Los Angeles, California 90025

T. Kim

Palos Verdes Peninsula High, Palos Verdes, California 90274

R. Buck

Westridge School for Girls, Woodland Hills, California 91105

E. Warfel

Palisades High School, Los Angeles, California 90272

T. Tasoff ${ }^{\mathrm{C})}$

Massachusetts Institute of Technology, Cambridge, Massachusetts 02139

J. Carmona

University of California, Los Angeles, California 90095

S. Skolnik ${ }^{\mathrm{c})}$

University of California, Berkeley, California 94720

L. Kim and D. Furlong

New Roads School, Santa Monica, California 90404

N. Gibson ${ }^{\text {d) }}$

University of Southern California, Los Angeles, California 90089

(Received 24 April 2006; accepted 29 September 2006)

We describe a successful alliance between a university and several high schools. The alliance is centered on a laboratory experiment constructed by students and faculty. The experiment involves sophisticated concepts and equipment not readily available in high schools. Much of the experiment is directly related to the science and mathematics learned in high school, with opportunities to extend their understanding by applying it to a research experience. The experiment is in plasma physics, but a similar alliance can be implemented in any area of science. Although the number of high school students affected by any one alliance is small, the impact is potentially large in the scientific life of a participating student or teacher. (C) 2007 American Association of Physics Teachers.

[DOI: 10.1119/1.2372470]

\section{INTRODUCTION}

For many years the American Association of Physics Teachers has supported the formation of college/university high school alliances. The Los Angeles Physics Teachers Alliance Group (LAPTAG) is one of these alliances. ${ }^{1}$ Since its formation in 1993, more than twenty schools have participated in the development of this alliance. In the early years 
LAPTAG encouraged communication between high school and college/university physics teachers by holding regular meetings, providing tours of laboratories at UCLA and other institutions, and discussion of curricular issues. LAPTAG also provided unique opportunities for student involvement in research projects.

In 1999 a group of high school teachers in collaboration with students and faculty from UCLA constructed a plasma physics device and began research on ion acoustic waves. Plasma physics was chosen for several reasons. The university faculty member (Gekelman) is an experimental plasma physicist and the director of a sizeable research program. Much of the device was constructed from spare parts and a vacuum chamber no longer in use. Some of the equipment was purchased through several small grants from the Department of Energy. Although the lab is located on the UCLA campus, it is for the exclusive use of high schools and thus is a high school plasma physics laboratory. The experiments enabled students to make contact with many of the concepts they were learning in high school and apply them in a fairly sophisticated context. Students have conducted experiments to measure the dispersion relation of ion acoustic waves in a low density, argon plasma. These experiments and others have engaged science students and encouraged them to enter physics-related careers.

\section{PHILOSOPHY OF LAPTAG}

There are two general ways to impact science education. One is a global approach undertaken by school boards and state legislatures. The other is a "village" approach undertaken by several educators interacting with a handful of students. We have adopted the second approach.

LAPTAG (Ref. 2) is a voluntary effort on the part of faculty and staff at UCLA, high school teachers, and high school students. Several junior college, and college students have also attended. All students that come are welcome. There are no entrance or exit exams, no certificates of completion, and no course credit. The students involved are interested in science; they are a self-selected group. Incoming students find out about LAPTAG from their high school science teacher, the LAPTAG website, ${ }^{1}$ or word of mouth. After attending, they realize they have access to laboratory equipment and specialized expertise that is not available at their high school. Most students return after coming for the first time and continue to attend about once a week for one or two years. Several still come even though they are now in college. There have been 111 high school students ${ }^{3}$ that have been attending regularly since 2000. LAPTAG alumni have become physics and engineering majors at top universities throughout the country. ${ }^{4}$

LAPTAG is not a project to develop new teaching methods or classroom curricula. It is not burdened with exit surveys or follow-up statistics to track the progress of alumni throughout their careers in college and graduate school. Many of the students that come to LAPTAG have excellent academic standing in their high schools and would go on to college in any case. What they get from LAPTAG is early advanced science exposure. If they wish to be challenged, they can do individual projects at any level. Several have done sophisticated experiments on their own during the summers and one reached the quarter finals of the Intel talent search.
The fact that LAPTAG is still in existence after 13 years speaks to its success. Students seek out LAPTAG and some drive 20 miles to participate. The first author has given several invited talks about LAPTAG at scientific meetings, 5 and students have made presentations at APS divisional meetings. ${ }^{6}$

\section{ION ACOUSTIC WAVES}

To date, the experiments have all involved ion acoustic waves. ${ }^{7}$ An ion acoustic wave is like a sound wave in air, in that it is compressional and involves density fluctuations. Ion acoustic waves, unlike sound waves, have an oscillating electric field, which arises because of charge separation due to the different masses of the ions and electrons. At low frequencies the dispersion relation looks very much like that of sound, $\omega / k=f \lambda=c_{s}$. The sound speed for an ion acoustic wave in the limit of cold ions is

$$
c_{s}=\sqrt{\frac{\gamma k T_{e}}{M_{I}}}=\left(9.8 \times 10^{5}\right) \sqrt{\frac{\gamma T_{e}}{\mu}},
$$

where $\mu=M_{I} / m_{p}$ is the ratio of the ion mass to that of a proton (40 for argon), $T_{e}$ is the electron temperature in electron volts $(1 \mathrm{eV}=11600 \mathrm{~K}), k$ is Boltzmann's constant, and $\gamma=c_{p} / c_{v}$ is the ratio of specific heats, which can be taken as unity for isothermal motion. The thermal conductivity of electrons is large enough for them to quickly adjust to the relatively low frequency wave. Because the electron temperature of a plasma is much higher than the temperature of air, ion sound waves usually travel hundreds of times faster than ordinary sound. At very high frequencies the dispersion of the sound wave is ${ }^{8}$

$$
\frac{\omega^{2}}{k^{2}}=\frac{\gamma K T_{e}}{M} \frac{1}{\left[1+\left(k / k_{D}\right)^{2}\right]},
$$

where $k=2 \pi / \lambda, k_{D}=\sqrt{4 \pi n e^{2} / k T_{e}}$ is the Debye wavenumber, $n$ is the plasma density, and $e$ is the magnitude of the charge of an electron. The dispersion relation for ion acoustic waves is shown in Fig. 1 for parameters typical of the LAPTAG experiment.

The ion plasma frequency or natural oscillation frequency of perturbed ions in a plasma is

$$
f_{\mathrm{pi}}=\sqrt{\frac{n e^{2}}{\pi M_{I}}}=210 \sqrt{\frac{n}{\mu}},
$$

for singly charged ions. As the wave frequency approaches the ion plasma frequency, the wave slows down and is damped. For these frequencies (the large wavenumber region of the curve in Fig. 1) the wavenumber eventually becomes independent of the frequency. The dispersion of these waves is interesting to beginning students because it is different from the dispersion of light and sound which they study in high school physics.

\section{THE EXPERIMENT}

The LAPTAG plasma device consists of a vacuum system capable of maintaining a vacuum of $10^{-6}$ Torr. A photograph of the LAPTAG plasma device and diagnostics is shown in Fig. 2. The vacuum is maintained by a small turbo-molecular pump, which is backed by a mechanical roughing pump. Incoming students learn about vacuum technology. Argon 


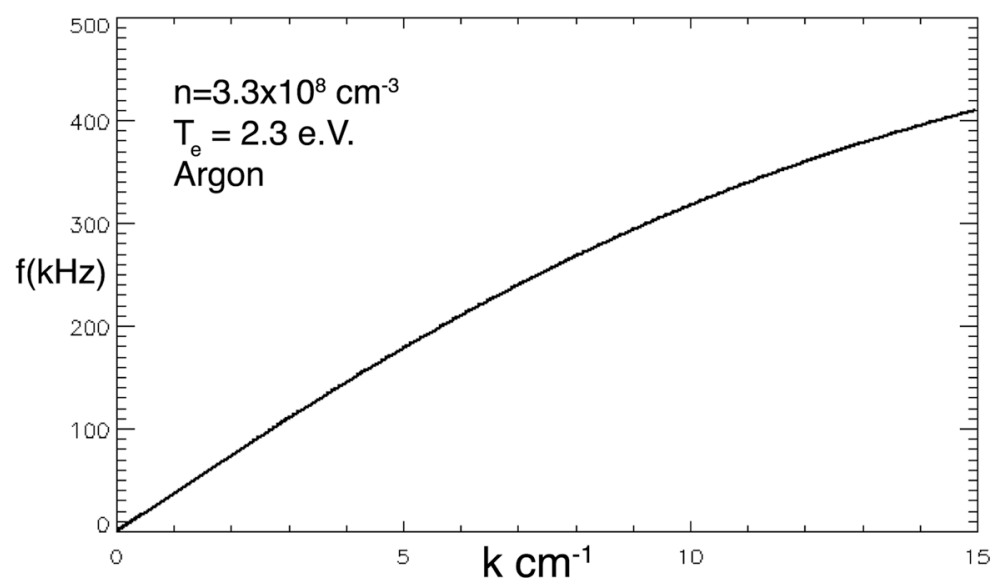

Fig. 1. Plot of Eq. (2), the dispersion relation for ion acoustic waves for typical conditions in the plasma. The wavenumber is in inverse centimeters.

gas is introduced into the chamber to a pressure of approximately $2.0 \times 10^{-4}$ Torr. The gas is ionized by using a twoturn copper strap antenna wound externally on a glass tube (4" inner diameter) mounted on one side of the main vacuum vessel and known as a helicon source. A 13.6 MHz commercial RF power supply drives the antenna. Variable capacitance vacuum capacitors are used along with the antenna inductance in a resonant LC circuit to match the $50 \Omega$ output impedance of the RF source. The two-strap antenna produces a plasma in the following manner. The azimuthal time varying current in the antenna induces a changing magnetic field along the axis of the glass tube that it is glued to with Kapton (high voltage insulating) tape. The changing magnetic field induces a polodial electric field in the gas, which breaks down and provides enough fast electrons to produce a plasma with density of order $n_{i}=n_{e} \simeq 3 \times 10^{8} \mathrm{~cm}^{-3}$. The percent ionization is low $\left(n_{e} / n_{\text {neutral }}=3 \times 10^{-5}\right)$. The antenna and plasma production provide examples of resonance in electri-

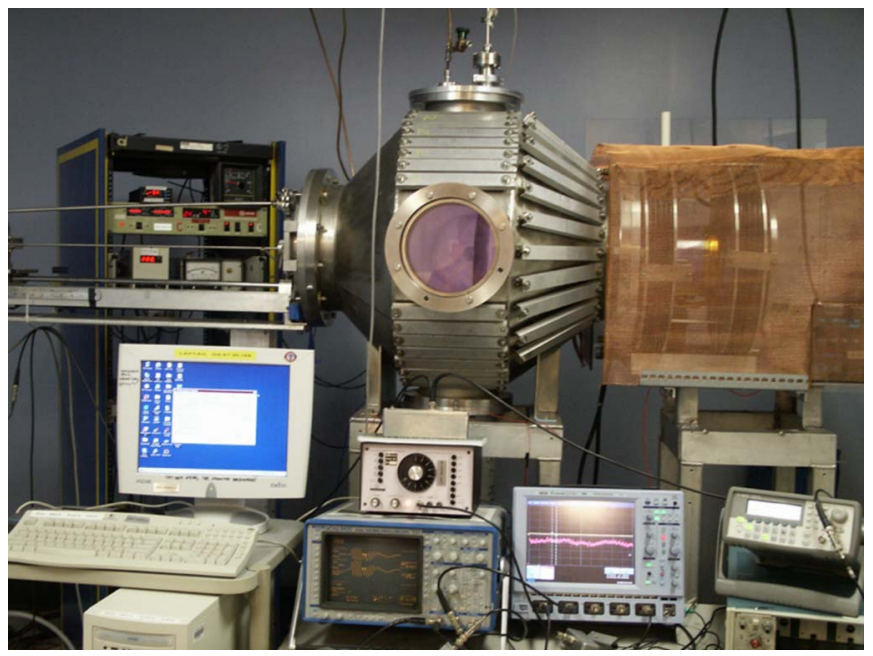

Fig. 2. LAPTAG plasma physics device. The plasma chamber is in the center (the plasma glows with a faint blue light) with the ion acoustic wave launch antenna visible in the right side of the center port. The RF source is enclosed in the screened area and the vacuum system gauges in the rack at the rear. The permanent magnets are held down by the aluminum channels. Magnetic coils are visible surrounding the glass chamber in the plasma source region, but they are not used in these experiments. cal circuits, Faraday's law, as well as the concept of ionization by fast electrons. Typical RF powers used are $40 \mathrm{~W}$ and the plasma is in a steady state. The external walls of the machine are covered with permanent magnets in a dipole configuration to confine the fast electrons. The resulting density of fast electrons is larger than it would be in the absence of the magnets and hence the plasma density is correspondingly higher. ${ }^{9}$ The magnetic field in the bulk plasma is nearly zero.

Although the plasma formation is somewhat complicated, running the device is simple. It involves bleeding in gas and turning on the RF source with minor tuning of the variable capacitor. Most students can do this procedure the first time they come to the lab. In this sense the understanding of plasma physics and the machine operation comes with participation, but is not required for participation.

The key elements in the ion acoustic wave experiments are the wave launching and detection scheme. The plasma density and density fluctuations due to the wave are measured by a Langmuir probe which is placed inside the plasma and connected to the outside by a coaxial vacuum feed-through. The wave is detected by measuring a signal to a biased metal disc inserted in the plasma. These simple electrode probes were invented by Langmuir ${ }^{10}$ in the 1920's and are still used. If the probe is biased positively with respect to the potential of the plasma, it will collect a current of electrons. If the bias is negative and the potential barrier around it is larger than the energy of the background electrons, the probe will collect an ion current. The fine details of the operation of a Langmuir probe, especially in the bias region where it collects a mixture of ions and electrons, are subtle. ${ }^{11}$ In these experiments the probe is usually biased to collect electron saturation current and the signal may be taken to be

$$
I_{\text {probe }}=n e A \sqrt{\frac{k T_{e}}{m_{e}}},
$$

where $A$ is the area of the probe and $m_{e}$ is the electron mass. The signal depends on both the electron temperature and plasma density. The electron temperature is independently derived from the phase velocity of ion acoustic waves. Although the ion temperature is not measured, it is known from work with similar RF sources to be of order $0.5-2.0 \mathrm{eV}$. $^{12}$ The ion temperature is too low to affect the current to the 


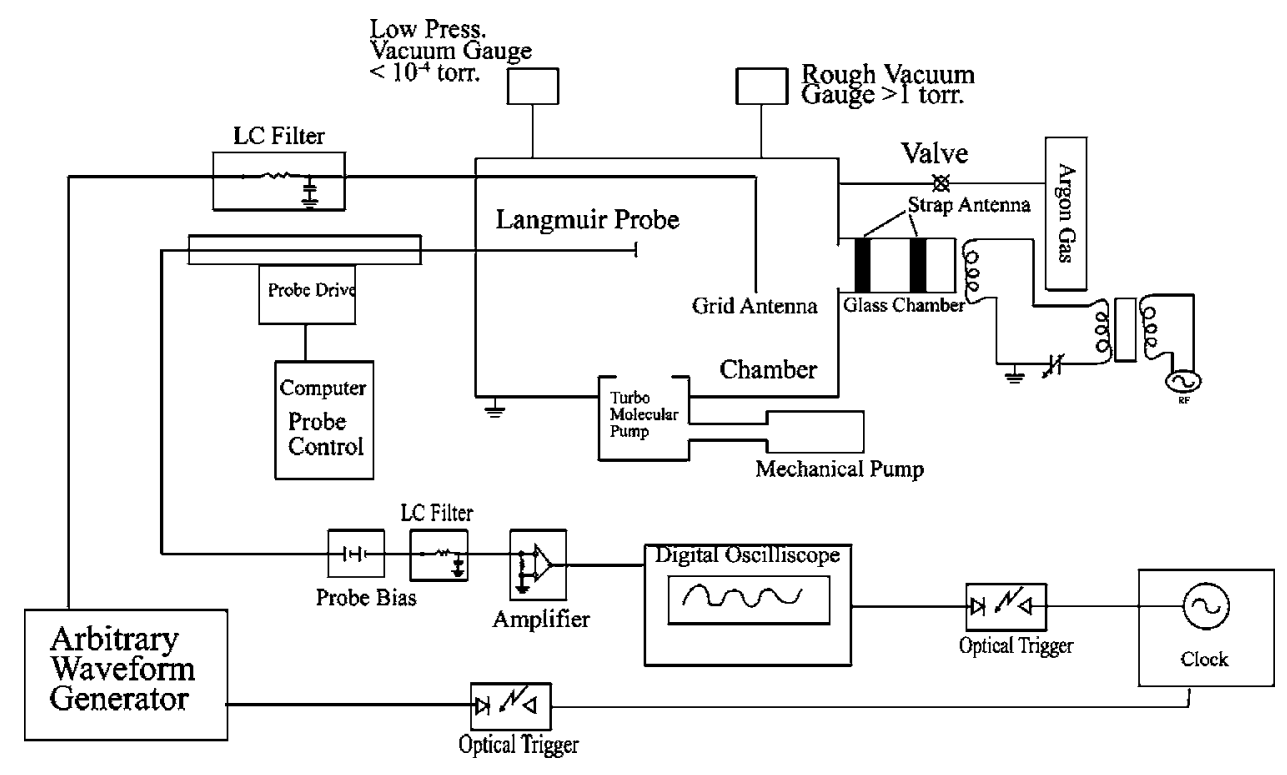

Fig. 3. Block diagram of the vacuum system, plasma source, wave launching, and detection scheme. The filters are used to prevent the $13.6 \mathrm{MHz}$ signal on the plasma-forming antenna from contaminating the low frequency wave signals.

probe or change the wave properties. Once the electron temperature is determined, the ion saturation current is used to estimate the plasma density from

$$
n=\frac{I_{\text {sat }}}{e A \sqrt{k T_{e} / M_{I}}},
$$

where $M_{I}$ is the mass of an argon ion. The waves are easily observed when the probe is biased to draw either ion or electron saturation current. When collecting electrons the probe bias must be greater than the plasma potential (the electric potential of the plasma with respect to the chamber walls) which is of order $+8 \mathrm{~V}$.

The Langmuir probe is easily constructed and students have made a number of them. The probe is a square of copper $2 \mathrm{~mm}$ on a side, which is connected to the inner conductor of a coaxial cable. The cable runs through a $0.25^{\prime \prime}$ diameter stainless steel tube that comes out of the vacuum system through a double sliding seal. The coaxial cable is attached to a modified BNC connector, which has an O-ring seal. The connector screws into a copper cylinder brazed to the stainless steel shaft. When the students assemble the probe, they can see what is necessary to keep air from leaking into the vacuum system through the probe and through seals as the probe moves. The probe is on a probe drive that was constructed by students. A brass cylinder at the end of a probe shaft is attached to a cart, which has a bearing that travels on a linear screw and is driven by a stepping motor. The stepping motor controller interface was programmed by one of the students. It has $0.5 \mathrm{~mm}$ positioning resolution along the axis of the device. The probe tip is biased with several batteries to $-66 \mathrm{~V}$ (with respect to the vacuum chamber wall) and current is drawn. The potential measured across a resistor in this circuit is proportional to the plasma density at the probe tip. The phase velocity of the wave is best determined by launching a tone burst of several cycles at the appropriate frequency $(50 \leqslant f \leqslant 400 \mathrm{kHz})$. A schematic diagram of the vacuum vessel and RF source and block diagram of the experimental wave launch and detection is shown in Fig. 3.
A free running square wave generator is used as an external trigger for the experiment, which is repeated every $2 \mathrm{~ms}$. The generator provides enough voltage to power a fiber optics triggering system designed by one of the UCLA research faculty and assembled and tested by a student. The electrical isolation provided by the fiber optics was a necessary step as ground loops plagued the early experiments. This system triggers an arbitrary function generator which outputs a tone burst (from 3 to 5 cycles at $1 \mathrm{Vpp}$ ) (volts peak-to-peak) to an antenna within the plasma. It also triggers a digital oscilloscope with an internal disk drive capable of storing the data. The wave-launching antenna is a circular copper mesh disk, $6^{\prime \prime}$ in diameter, attached to a coaxial cable much like the one for the Langmuir probe. When the potential of the antenna with respect to the walls of the device is modulated, charged particles are attracted or repelled from the antenna at the frequency of modulation. This modulation causes small density compressions and rarefactions and triggers the wave in analogy to how a sound wave in air is generated by a vibrating drum. Both sound waves and ion acoustic waves are longitudinal waves. The difference is that the ion acoustic wave carries fluctuations of electric potential as well as density fluctuations. A large antenna is used to generate a plane wave.

The antenna shaft also goes through a sliding seal so that the position of the antenna with respect to the plasma source can be varied. The probe is biased positively to collect the electron saturation current. The probe signal is sent to a high input impedance amplifier with variable input and output cutoff frequencies. The signal is filtered to remove the 13.6 MHz waves from the source as well as other plasma noise outside of the range of ion acoustic waves. Because we are only interested in density fluctuations, the probe signal is $\mathrm{AC}$ coupled to the amplifier. For a given wave frequency the probe is moved in small steps and the signal, averaged over 1000 bursts, is stored. It is necessary to average over many shots because of the noise inherent in RF produced plasmas. Figure 4 is a space-time diagram which shows the received 


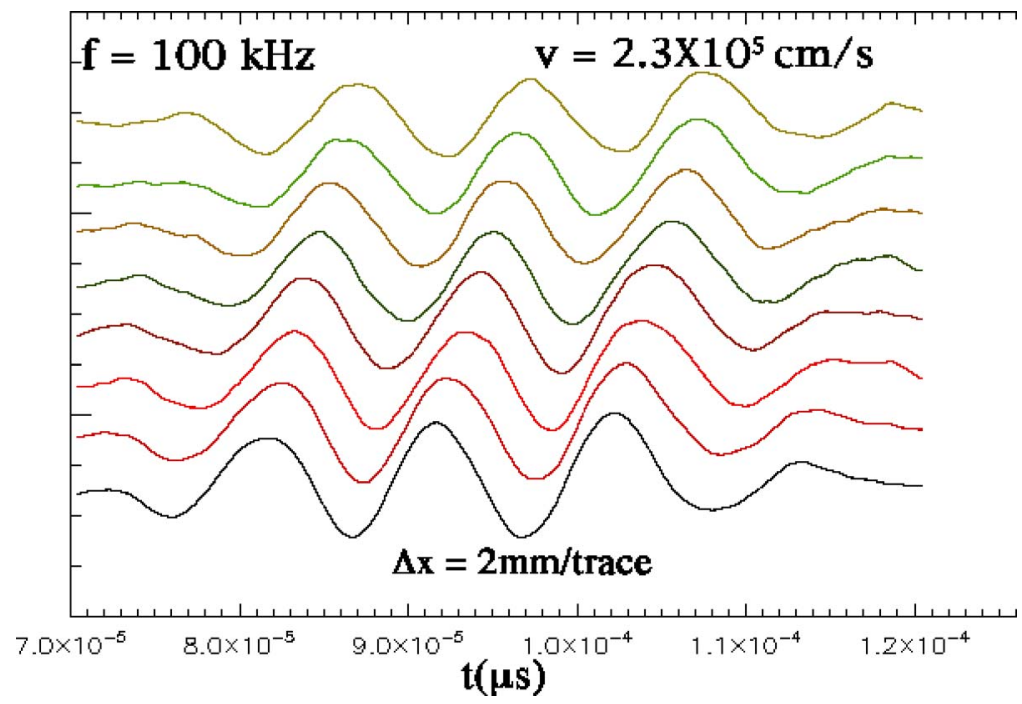

Fig. 4. Data from digitally stored data of the received ion acoustic tone burst. The data is density fluctuations of the wave at eight axial locations spaced by $2 \mathrm{~mm}$. The phase velocity is the ion acoustic speed. The distances increase away from the antenna located at $\Delta x=-17.5 \mathrm{~cm}$. The curves have been displaced vertically to illustrate the phase propagation.

signal at eight probe locations separated by $2 \mathrm{~mm}$. An alternate way of viewing the space-time behavior is a surface plot, Fig. 5. By following a point of constant phase, or equivalently the slope in Fig. 5, we arrive at the phase velocity of the ion acoustic wave, $v=d x / d t=2.3 \times 10^{5} \mathrm{~cm} / \mathrm{s}$. The electron temperature may be estimated from Eq. (1) and is $T_{e}=2 \mathrm{eV}$ or $24000 \mathrm{~K}$.

The dispersion relation of the ion acoustic wave is measured as follows. A five-cycle tone burst at a given frequency is applied to the launch antenna shown in Figs. 2 and 3. The
Langmuir probe is movable in a line parallel to the normal to the antenna and is biased to collect electron saturation current. This probe detects the wave. The acquired signal goes to a tuned $(10 \mathrm{kHz}-1 \mathrm{MHz})$ amplifier and then to a digital oscilloscope where it is averaged over a thousand shots at each position to eliminate plasma and amplifier noise, and is low pass filtered to remove $\mathrm{rf}$ noise. The probe is then moved $5 \mathrm{~mm}$ and a second average is acquired. The phase shift at each frequency is used to determine the wavelength. A plot

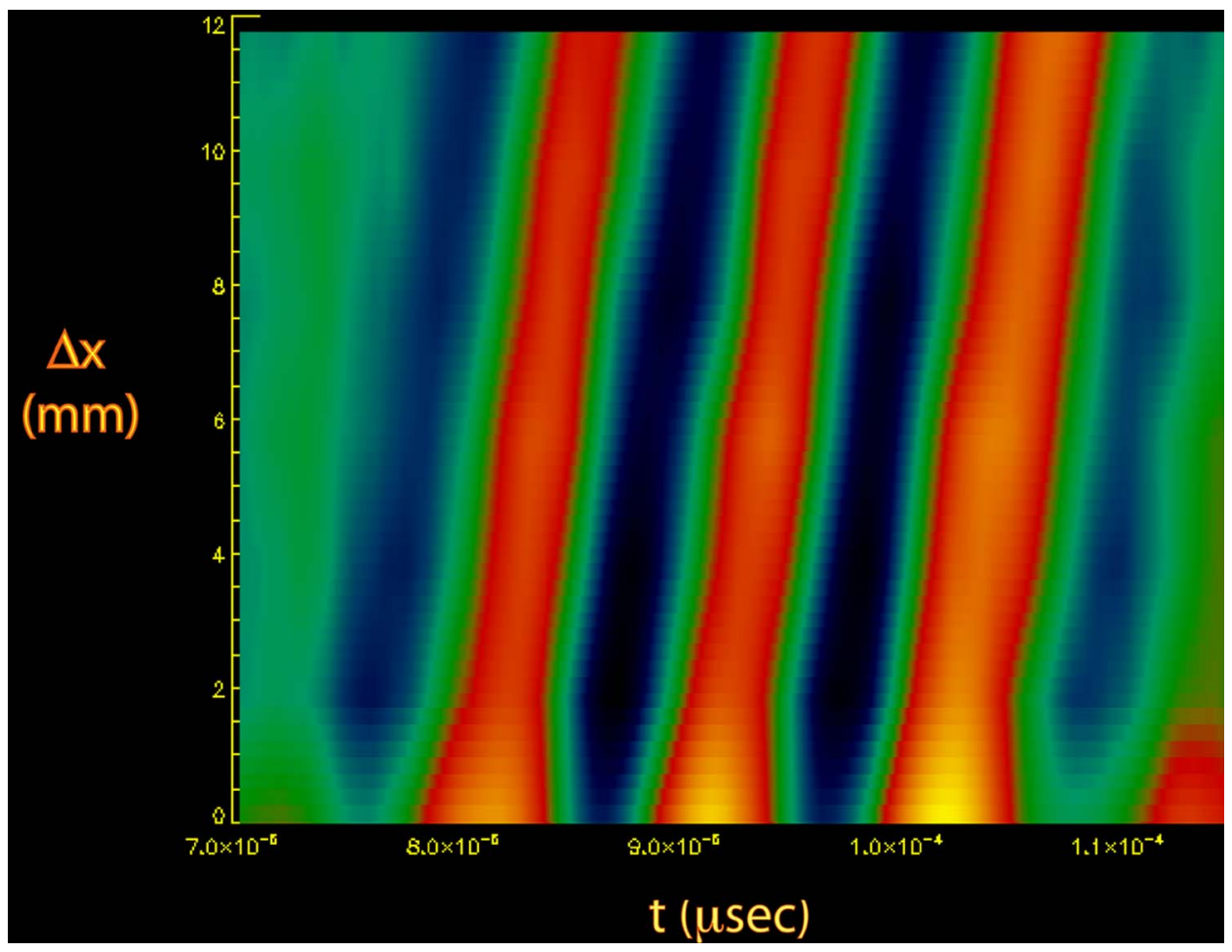

Fig. 5. Space-time diagram of an ion acoustic tone burst. The inclined burst shows three cycles of the ion acoustic wave. The time is in microseconds and the ordinate is the distance in millimeters. 




Fig. 6. Measured dispersion relation of the ion acoustic wave and best linear fit. Note the turnover of the data close to the ion plasma frequency, which is approximately $420 \mathrm{kHz}$.

of the dispersion relation is shown in Fig. 6. The $\omega$ versus $k$ relation is linear until $f \simeq 420 \mathrm{kHz}$, which corresponds to a density of $n=1.6 \times 10^{8} \mathrm{~cm}^{-3}$. The wave phase velocity is given by the slope of the linear portion of the dispersion curve or $v_{\text {phase }}=5.4 \times 10^{5} \mathrm{~cm} / \mathrm{s}$. This value is larger than that derived from Fig. 4, but the results of the experiments shown in Fig. 4 were done at different neutral pressures and $\mathrm{rf}$ source powers.

The damping of the wave as the plasma frequency is approached has been observed in the LAPTAG experiment, but the interpretation involves collisionless (Landau) damping ${ }^{13}$ of the wave as well as collisions with neutral atoms. ${ }^{14}$ The mathematics is too complicated for high school students and only a general explanation is possible. The slowing down of the wave comes from the dispersion relation in Eq. (2). In the experiment the time of flight of a four-cycle tone burst is measured as a function of frequency. The experiment is sensitive enough to measure the slowing down of the wave as shown in Fig. 7. The argon plasma density was approximately $3.3 \times 10^{8} \mathrm{~cm}^{-3}$ and the electron temperature about $2.3 \mathrm{eV}$. The measurement was repeated ten times at several locations to determine the experimental error. The solid curve is the theoretical wave delay determined by calculating the group velocity of the wave, $d \omega / d k$, from Eq. (2). The temporal offset due to the distance from the antenna to the receiving probe is subtracted.

\section{GENERAL PHYSICS CONCEPTS- EDUCATIONAL VALUE}

The greatest strength of this program is the interaction of high school students with scientists and faculty. High school students rarely get this kind of access and exposure to scientists who are active researchers.

The LAPTAG experience has exposed participating students to many general-physics concepts that have been reinforced by applications. Because the plasma lab experience is less constrained by time and content than traditional courses, students can spend as much time as they like on physics topics that interest them. Students learn about vacuum systems (they are usually surprised that under vacuum we do not need bolts to hold flanges onto the machine). They use the ideal gas law to calculate the number of atoms of argon in the vacuum chamber at a certain pressure and temperature. They calculate the speed of an ion acoustic wave by changing the location of the probe a known distance and measur-

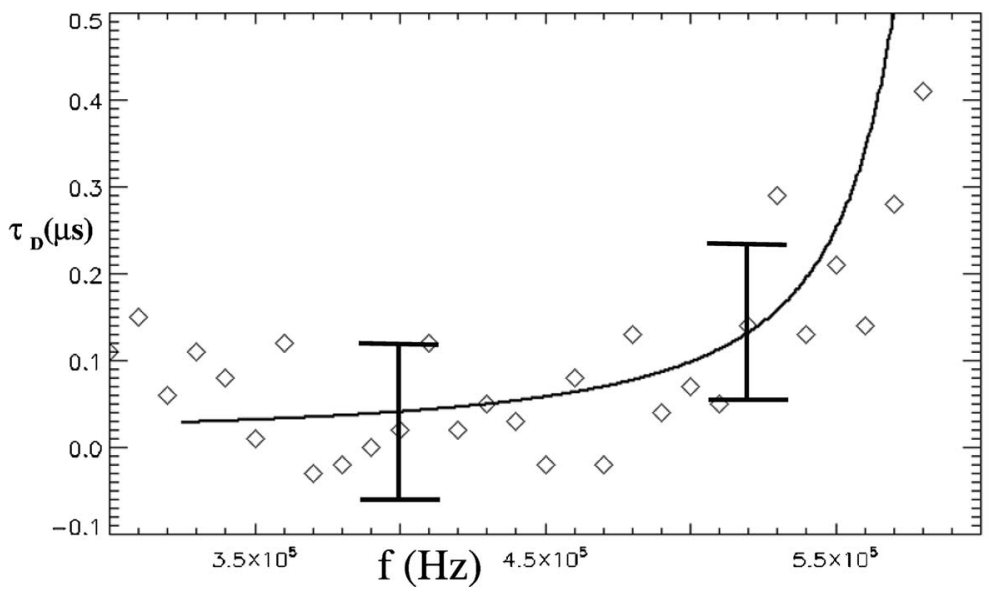

Fig. 7. The arrival time of the center of an ion acoustic tone burst as a function of wave frequency. The points on the left correspond to the linear part of the dispersion curve (Fig. 6). The solid line is the theoretical curve for the delay. The vertical bars denote the measurement error. 
ing the signal time delay using an oscilloscope. They use the concept of phase shift for experimental purposes. Students learn about Faraday's law when they have to determine how to reduce the external interference that they are detecting in their wave signal. The electronics involved in making the plasma and detecting the wave in the plasma include simple circuits, resonant frequencies, impedance matching, and filtering.

The equipment in the lab excites students and motivates them to learn. Students explore and discuss ion pressure gauges, rf power supplies, O-rings, oscilloscopes, signal generators, filtered amplifiers, and machined parts. This experience is important in maintaining interest in and preparing for careers in science. In addition to the experiments, lectures have been given on plasma physics, Fourier analysis, noise reduction, and signal correlation. Students have been introduced to the higher mathematics necessary for describing some of the concepts surrounding waves in plasmas. Students have learned to use powerful software tools for data analysis such as PV-wave or IDL.

By discussing how a wave travels through a plasma, students reinforce and extend what they have learned about waves in their high school physics courses, for example, wavelength, period, frequency, dispersion, and velocity. These terms often lack significant meaning for the students because they have limited, if any, experience with these terms. In the plasma lab students participate in the discussion of research questions and results in a way that provides meaningful context for what they are learning in high school. In this way, they move from a superficial knowledge to a comprehensive understanding of waves and the underlying physics. ${ }^{15}$

\section{UNIVERSITY-HIGH SCHOOL ALLIANCES}

LAPTAG was created so that a university could interact strongly with high schools to strengthen the high school science educational process. Many of the teachers in the group have been in education for decades, and have developed innovative curricula and effective demonstration materials. What we have in common is a dedication to science and a desire to broaden the educational experience that high schools offer. The high school teachers in this alliance have had much experience in innovative projects, science fairs, curriculum development and the like. Their experience has led to the following observations:

1. Students of all backgrounds are "turned on" by projectoriented tasks. They come alive when asked to work with high-tech equipment, particularly when they understand how it works.

2. The need for relevance in high school science education is tremendous. The average student is bored and sees no reason to pursue the study of science. Parents do not relate the importance of the study of science to their children and teachers do little to steer them in that direction. A relevant topic, such as the study of plasmas and energy science, is a strong motivator. In addition, it would bring them into contact with a branch of science they have not seen in class.

3. Many of the jobs emerging in today's society are technology driven. Few students have seen any of the tools used in such endeavors. Working on a plasma physics device in an active laboratory would be a thrill for the students in our secondary schools and would help bridge the technology gap.

These statements and suggestions are based on anecdotal evidence and not on rigorous surveys used by those wishing to change the curricula of a school or a school district.

LAPTAG was formed as a result of an NSF sponsored workshop on collaborations between universities and high schools held at Cal Poly Pomona in 1994. Most of the alliances formed at that time have vanished. We have learned, through experience, that the "secret" to the success and longevity of our alliance rests on four key points.

1. The identification of a project that high school students and faculty can build and manage provided a focus point for the collaboration. The LAPTAG Plasma Lab project was closely aligned with the interests of the university mentor. Without enthusiasm for the study of plasmas and the building of this machine at the university, the project could not have succeeded. The experiment had the following qualities: (a) It could be related to what students were learning in high school science. (b) It was visual, that is, few hidden parts. (c) It does not always work, which means that there is some effort involved in collecting data. (d) There is some novelty involved in the study of plasmas.

2. All participants are self-selected. There is no application process. We encourage a range of students from freshmen to high school seniors. This range of participants encourages peer collaboration and the development of a supportive environment for asking questions and discussing concepts.

3. There is no fixed schedule for working in the lab other than we always try to meet once a week on Saturday mornings at 9:30 AM. During the summer high school teachers and students drop in several days a week. Once we decide to do an experiment, we make any configuration changes to the machine and build any necessary instruments. We work on our experiments when and while the machine is working well.

4. We have found that focusing on keeping the machine running and developing interesting experiments has provided relevant learning opportunities for all participants. There is no need for a specified curriculum. When a topic comes up that needs discussion, we schedule a lecture series to address the new concepts.

The use of plasma physics as a vehicle is a consequence of the specialization of the first author. Any branch of physics would work as well. The ingredients are a college professor with expertise in some area of interest, some spare equipment, and a relationship (which must be cultivated) with nearby high schools. A second necessary ingredient is one or more dedicated high school teachers. Generally there is no lack.

\section{ACKNOWLEDGMENTS}

The authors happily acknowledge the support of the Department of Energy Office of Fusion Science for several grants used to help fund the device construction and the ongoing educational outreach support of the Basic Plasma Science Facility at UCLA, which is funded by a cooperative agreement between the National Science Foundation and the 
Department of Energy. We also happily acknowledge the support that the UCLA Vice Chancellor for Research, Roberto Peccei, has given over the years.

${ }^{\text {a) }}$ Former address: Milken Community High School, Los Angeles, California 90049

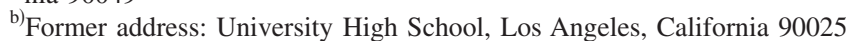

${ }^{c}$ Former address: Sherman Oaks Center for Enriched Studies, Los Angeles, California 91335

${ }^{d)}$ Former address: Crossroads School, Santa Monica, California 90404

${ }^{1}\langle$ http://coke.physics.ucla.edu/laptag $\rangle$.

${ }^{2}$ W. Gekelman, "Laptag: A physics outreach program at UCLA," APS News 11, 5 (2002).

${ }^{3}$ The high schools include Chaminade High School, West Hills; University High School, West Los Angeles; Sylmar High, Sylmar; Crossroads High School, Santa Monica; Louisville High School, Woodland Hills; Harvard Westlake, North Hollywood; Birmingham High School, Van Nuys; San Marino High, Pasadena; New Roads School, Santa Monica; Notre Dame High School, Sherman Oaks; Fairfax High School, Hollywood; Milken Community High, Los Angeles; Peninsula High School, Palos Verdes; California State University, Los Angeles; Palisades High School, Pacific Palisades; Winward High, Culver City; Bridges Academy, Sherman Oaks; Buckley School, Sherman Oaks; Loyola High, Los Angeles; Compton High School, Compton; Monroe High School, Burbank. One student was home schooled.

${ }^{4}$ The universities include Carnegie-Mellon University, California Institute of Technology, Massachusetts Institute of Technology, University of Minnesota, and the University of California campuses at Los Angeles, Riverside, and Berkeley.

${ }^{5}$ W. Gekelman, "Laptag, the Los Angeles Physics Teachers Alliance Group," LI2.001, APS Division of Plasma Physics, Long Beach, CA, Oct. 30, 2001; W. Gekelman, "Laptag: A Physics Outreach Program at UCLA," T6.002, APS April Meeting, Philadelphia, PA, April 8, 2003.

${ }^{6}$ The following posters were given at the APS Division of Plasma Physics Meeting, Oct. 30, 2000, Long Beach, CA. W. Layton, W. Gekelman, J. Wise, N. Rodriguez (Santa Monica Community College), S. Cooperman (Milken High School), R. Griffen (Campbell Hall High School), J. Altonji (Sylmar High School), F. Carrington (Grant High School), B. Coutts
(Van Nuys High School), and K. Coutts (Royal High School), "Encouraging high school students to consider physics related careers;" M. Buck (Chaminade High School), J. Wise, B. Baker, J. Altounji (Sylmar High School), R. Buck, C. Spahn (Monroe High School), W. Gekelman and P. Pribyl, "Construction of a high school plasma laboratory;" R. Buck, J. Wise, N. Gibson, M. Buck, W. Gekelman, E. Wetzel (Louisville High School), C. Wetzel (Loyola High School), and C. Moynihan, "Ion acoustic waves, A high school plasma experiment;" P. Hsu (Compton High School), B. Baker, J. Wise, M. Buck, R. Buck, and W. Gekelman, "Characteristics of the LAPTAG high school plasma;" J. A. Wise (New Roads High School), M. Buck (Chaminade High School), W. Gekelman, R. Buck, C. Spahn, C. Walker (Louisville High School), and B. Layton, "Using plasma physics to enhance the high school physics curriculum."

${ }^{7}$ I. Alexeff and R. V. Neidigh, "Observations of ion sound waves in plasmas," Phys. Rev. Lett. 7, 223-225 (1961).

${ }^{8}$ N. Krall and A. Trivelpiece, Principles of Plasma Physics (McGraw-Hill, New York, 1973), pp. 389-391.

${ }^{9}$ K. N. Leung, T. K. Samec, and A. Lamm, "Optimization of permanent magnet plasma confinement," Phys. Lett. 51A, 490-492 (1975).

${ }^{10} \mathrm{H}$. M. Motts-Smith and I. Langmuir, "The theory of collectors in gaseous discharges," Phys. Rev. 28, 727-763 (1926); I. Langmuir, "Collected works of Irving Langmuir," edited by G. Suits (Pergamon Press, New York, 1961), Vol. 4.

${ }^{11}$ N. Herskowitz, "How Langmuir probes work," in Plasma Diagnostics, edited by O. Auciello and D. Flamm (Academic Press, New York, 1989), Vol. 1.

${ }^{12}$ M. G. Blain, J. E. Stevens, and J. R. Woodworth, "High resolution submicron retarding field energy analyzer for low-temperature plasma analysis," Appl. Phys. Lett. 75, 3923-3925 (1999).

${ }^{13}$ F. F. Chen, Introduction to Plasma Physics and Controlled Fusion (Plenum Press, New York, 1984), 2nd ed., pp. 240-256.

${ }^{14}$ S. Brown, Basic Data of Plasma Physics (Wiley, New York, 1959).

${ }^{15}$ Much research has been done in the area of conversation/discourse and student learning. See for example, J. Roschelle, "Learning by collaborating: Convergent conceptual change," J. Learn. Sci. 2, 235-276 (1992); K. Hogan, B. Nastasi, and M. Pressley, "Discourse patterns and collaborative scientific reasoning in peer and teacher-guided discussions," Cogn. Instruct. 17, 379-432 (1999).

\section{BOSE}

"There are various ways that fame comes to a scientist. For Satyendranath Bose it was asking Albert Einstein to run interference for him. Eventually his name was linked with Einstein's in both a statistical method of dealing with quantum particles, called BoseEinstein statistics, as well as the particular state of matter known as the Bose-Einstein condensate. In addition, Bose had a class of particles named after him: the boson. As this example illustrates, Einstein's scientific influence was telling."

John S. Rigden, Hydrogen: The Essential Element (Cambridge University Press, Cambridge, Massachusetts, 2005), p. 234. 